\title{
Leser-Trelat sign in mother and daughter with breast cancer
}

\author{
HENRY T LYNCH,* RAMON M FUSARO, $\dagger$ JUDITH A PESTER, $\ddagger$ AND \\ JANE F LYNCH*
}

From * the Department of Preventive Medicine, Creighton University, Omaha, Nebraska 68178; $\dagger$ Creighton-Nebraska Universities Health Foundation Dermatology Program, Omaha, Nebraska 68105; and $\ddagger$ the Department of Pathology, University of Nebraska Medical Center, Omaha, Nebraska 68105, USA

SUMMARY The Leser-Trelat sign is the sudden appearance and rapid increase in size and number of seborrhoeic keratoses in association with cancer. Twenty cases of this unusual phenomenon have so far been reported in the world literature. More than half involve adenocarcinomas and none was shown to be familial. We report a unique example of a 41-year-old black female and her 74-year-old black mother, both of whom have demonstrated classical clinical-pathological evidence of the Leser-Trelat sign and adenocarcinoma of the breast. The remainder of the family history was negative for cutaneous lesions and cancer. The aetiological and pathogenetic significance of the Leser-Trelat sign in association with carcinoma of the breast in this mother and daughter remains obscure.

The criteria for the Leser-Trelat sign are the sudden appearance and rapid increase in size and number of seborrhoeic keratoses in association with visceral cancer. ${ }^{1}$ Pruritus may or may not be associated and the sign may or may not disappear after removal of the cancer. Dantzig ${ }^{2}$ reviewed the medical literature on the Leser-Trelat sign and found that between 1891 and 1973 a total of ten cases had been reported. Sperry and Wall ${ }^{1}$ found an additional nine cases, including a case report of their own. More recently, Lee $e t \mathrm{al}^{3}$ reported an additional patient with bile duct adenocarcinoma, pure red blood cell aplasia, and the Leser-Trelat sign, thus making 20 documented cases up to 1980 . The majority of cancers were adenocarcinomas. There was not a single example of a positive family history of the Leser-Trelat sign. Nevertheless, from our own experience, we have observed the family history to be often seriously neglected in the study of patients with cancer. ${ }^{4}$

The purpose of this report is to describe what we believe to be the first familial example of the LeserTrelat sign as shown by its occurrence in a mother and daughter, each of whom had verified adenocarcinoma of the breast.

Received for publication 20 July 1981

\section{Case reports}

THE DAUGHTER

This 41-year-old, single, black female had a biopsy of the left breast diagnosed as a fibroid adenoma 10 years ago. She does not drink alcohol, but has smoked cigarettes for 20 years. She has been in good physical health.

Six weeks before admission (July 1980), she noted nipple retraction, breast asymmetry, and a large mass under the right nipple. She underwent an incisional biopsy and subsequent modified mastectomy for infiltrating ductal carcinoma stage III. Thirty-four lymph nodes were examined, of which 20 were positive, including 16 at level 3 . The mass was $4 \mathrm{~cm}$ in size and there was evidence of lymphatic and perineural invasion (T2, N2, M0). Oestrogen receptors were negative.

She received local postoperative irradiation of the right chest wall, internal mammary nodes, axilla, and supraclavicular region with a total dose of 5000 rads in 5 weeks. One week after discharge, she was placed on a low dose chemotherapy protocol consisting of cyclophosphamide, methotrexate, and 5-fluorouracil. After completion of $x$-ray therapy, the dosages of the drugs were increased to their present standard levels.

She denies that she had any skin lesions with the appearance of seborrhoeic keratoses before about 
6 months before the diagnosis of her breast cancer. At that time, a sudden showering of lesions appeared on her anterior and posterior neck, chest, and back, with further extension down to her abdomen. The lesions consisted of many small 1 to $4 \mathrm{~mm}$ brown to black lesions, which were usually macular, but were occasionally slightly papular (fig 1A, B). They reached their full size and complement in about 2 to 3 months after her mastectomy and they have not shown any further extension nor have they shown eivdence of regression up to the present time (8 months after the mastectomy).

\section{THE MOTHER}

This 74-year-old black female, mother of the above patient, underwent a total mastectomy at the age of 67 for an infiltrating ductal carcinoma of the right breast. She was treated by radiation therapy.

She had a history of recurrent diverticulitis and oesophageal diverticulum, but was otherwise in good health. She drank alcohol only rarely and did not smoke.
She had several seborrhoeic keratoses on her upper trunk for a prolonged period before the occurrence of her cancer. The exact date of their onset was not known, but it may have been as long as 10 years before the breast cancer diagnosis. About 2 to 3 months before the diagnosis of her breast cancer and for a couple of months after her mastectomy, she experienced a continuous showering of dark lesions which covered much the same anatomical distribution as those in her daughter, namely the anterior and posterior neck, chest, and back. These lesions have not shown any further progression nor have they regressed in size or number. At the present, she has numerous brown to black, soft, greasy, papular, domed to flat lesions with a velvety to fine verrucous surface up to almost $2 \mathrm{~cm}$ in diameter (fig $1 \mathrm{C}, \mathrm{D}$ ).

The remainder of the family history was unremarkable. Specifically, we found no further evidence of cancer or cutaneous lesions in the maternal and paternal lineage of the mother nor in the paternal lineage of the daughter.

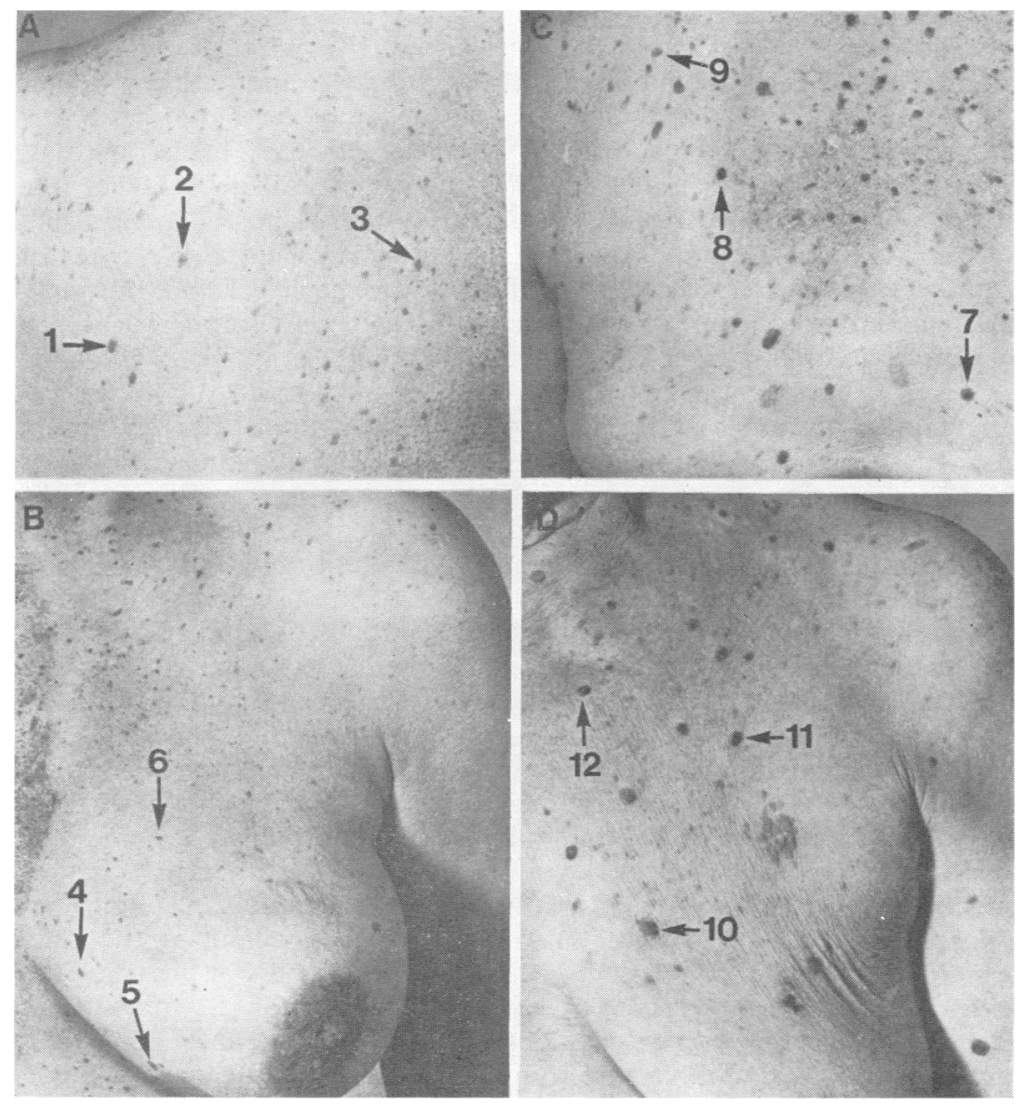

FIG 1 The left back and front, respectively, of $(A, B)$ the 41 year-old daughter, and $(C, D)$ the 74-year-old mother. The numbers indicate the biopsy sites of the seborrhoeic keratoses. 


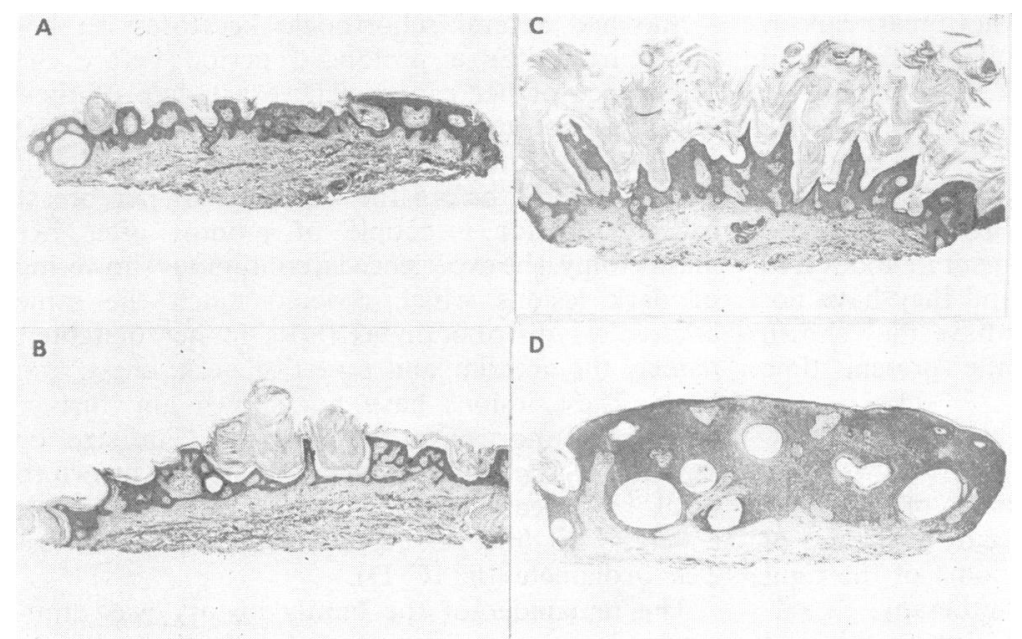

FIG 2 (A) Seborrhoeic keratosis No 3 under low power shows an acanthotic and hyperkeratotic pattern. (B) Seborrhoeic keratosis No 5 is hyperkeratotic and adenoid in configuration.

(C) Seborrhoeic keratosis No 11 is a hyperkeratotic type.

(D) Seborrhoeic keratosis No 12 has an acanthotic pattern. (Original magnification, $\times 2 \cdot 5$. Haematoxylin and eosin.)

\section{Histology}

On each patient, six lesions (daughter: fig $1 \mathrm{~A}, \mathrm{~B}$, lesions 1 to 6; mother: fig $1 \mathrm{C}, \mathrm{D}$, lesions 7 to 12) were randomly selected for histological verification. All of these were seborrhoeic keratoses; however, the mother and daughter showed different histological patterns.

The daughter showed several types of seborrhoeic keratoses which were not as thick histologically as those in the mother. This was true not only histologically, but also clinically. Four of the skin lesions showed a combination pattern of hyperkeratosis and acanthosis (fig 2A). The other two lesions showed a combination pattern of the hyperkeratotic and adenoid types (fig 2B).

The seborrhoeic keratoses of the mother were predominantly of the hyperkeratotic type. Four of these lesions showed a hyperkeratotic configuration in that there was hyperkeratosis and papillomatosis (fig 2C). One of these lesions showed a combination of the hyperkeratotic and acanthotic patterns. The twelfth lesion was exclusively of the acanthotic type. In this latter lesion, there was evidence of thick tracts of epidermal cells that extended into the dermis (fig 2D). Interspaced among these basaloid cells were cystic inclusions which contained keratin material.

Even though the daughter and mother both had seborrhoeic keratoses, there was some variation in the type of lesion. The mother essentially showed only a hyperkeratotic or acanthotic pattern. The daughter showed a combination of configurations which included the hyperkeratotic, acanthotic, and adenoid types. These differences must be considered with respect to the ages of the two patients and the number of years these lesions have been present on each patient.

\section{Discussion}

Of the 20 patients with the Leser-Trelat sign cited in the literature, 14 had adenocarcinoma. ${ }^{3}$ The organs involved were stomach (five cases), large bowel (three cases), pancreas, ovary, breast, uterus, prostate, and bile duct (one case each). Hence, the gastrointestinal tract was the most common end organ system. Other lesions included two cases of mycosis fungoides (one case being Sezary syndrome), one of hepatoma (associated with porphyria cutanea tarda), one of squamous cell carcinoma of the lung, one of lymphoma, and an unspecified tumour. ${ }^{3}$

Little is known about the pathogenetic mechanism that relates the rapid onset of seborrhoeic keratoses with systemic cancer in other organs This could be the result of an as yet unknown but unusual carcinogenic exposure, which not only leads to visceral cancer, but also provides the necessary conditions for the inordinate susceptibility to rapid development of multiple seborrhoeic keratoses of the skin. Another possibility is that the Leser-Trelat sign may represent a unique type of cancer-associated genodermatosis, ${ }^{5}$ albeit one in which the skin signs may be either constant or transitory, regressing in some cases when all of the tumour has been removed.

Several possible explanations for these associations exist. (1) In a fraction of cases, the susceptibility to the Leser-Trelat sign is genetic and is the only component which is hereditary, but requires the presence of systemic cancer for its phenotypic expression. (2) In our patients, we may be dealing with a rare form of heritable breast cancer having a 
peculiar antigen which triggers an immunological response manifested in the skin as the Leser-Trelat sign. In this example, the susceptibility to the Leser-Trelat sign may not represent a susceptibility to cancer per se. Rather, it may be a potentiality or latent state which can only be expressed in the presence of a tumour which has achieved a critical stage of development. (3) Both the susceptibility to the Leser-Trelat sign and to breast cancer may be genetically determined by closely linked genes or by a single pleiotropic gene. Finally (4) the LeserTrelat sign may have multiple aetiologies.

The hypothesis of genetic influence may account for only a small fraction of the appearances of the Leser-Trelat sign. Indeed, it is likely that at our present state of knowledge, the majority of occurrences are not genetic. Our single case report of a mother and daughter with breast cancer and the Leser-Trelat sign will not settle this issue. We will need keen clinical observations of cutaneous signs coupled with detailed family histories in patients with all varieties of cancer. These concerns are stressed because cutaneous signs and family history are often ignored or given little regard in patients with visceral cancer. ${ }^{45}$ In the case of seborrhoeic keratoses, this becomes even more problematical since these lesions commonly occur in elderly patients as do most varieties of cancer. However, as already mentioned, the differentiating feature is the history of rapid evolution in size and number of seborrhoeic keratoses in temporal association with visceral cancer.

In conclusion, we admonish our readers to exercise caution in the interpretation of the LeserTrelat sign associated with breast cancer in the mother and daughter. Seborrhoeic keratoses are exceedingly common in older age groups as is breast cancer. Therefore, the possibility that this phenomenon could be fortuitous cannot be excluded.

\section{References}

1 Sperry K, Wall J. Adenocarcinoma of the stomach with eruptive seborrheic keratoses: the sign of Leser-Trelat. Cancer 1980;45:2434-7.

2 Dantzig PI. Sign of Leser-Trelat. Arch Dermatol 1973; 108:700-1.

3 Lee CH, Clark AR, Thorpe ME, Mackie BS, Firkin FC. Bile duct adenocarcinoma with Leser-Trelat sign and pure red blood cell aplasia. Cancer 1980;46:1657-60.

4 Lynch HT, Follett KL, Lynch PM, Albano WA, Mailliard JA, Pierson RL. Family history in an oncology clinic: implications for cancer genetics. JAMA 1979;242: 1268-72.

5 Lynch HT, Fusaro RM. Cancer-associated genodermatoses. New York: Van Nostrand Reinhold (in press).

Requests for reprints to Professor H T Lynch, Department of Preventive Medicine, Creighton University, 2500 California Street, Omaha, Nebraska 68178 , USA. 